Original paper

\title{
The TOPAS tool for particle simulation, a Monte Carlo simulation tool for physics, biology and clinical research
}

\author{
Bruce Faddegon $^{\mathrm{a}, *}$, José Ramos-Méndez ${ }^{\mathrm{a}}$, Jan Schuemann ${ }^{\mathrm{b}}$, Aimee McNamara ${ }^{\mathrm{b}}$, Jungwook Shin ${ }^{\mathrm{b}}$, \\ Joseph Perl ${ }^{\mathrm{c}}$, Harald Paganetti ${ }^{\mathrm{b}}$
}

${ }^{a}$ Department of Radiation Oncology, University of California San Francisco, San Francisco, CA, USA

${ }^{\mathrm{b}}$ Massachusetts General Hospital and Harvard Medical School, Boston, USA

${ }^{\text {c }}$ SLAC National Accelerator Laboratory, Menlo Park, USA

\section{A B S T R A C T}

Purpose: This paper covers recent developments and applications of the TOPAS TOol for PArticle Simulation and presents the approaches used to disseminate TOPAS. Materials and methods: Fundamental understanding of radiotherapy and imaging is greatly facilitated through accurate and detailed simulation of the passage of ionizing radiation through apparatus and into a patient using Monte Carlo (MC). TOPAS brings Geant4, a reliable, experimentally validated MC tool mainly developed for high energy physics, within easy reach of medical physicists, radiobiologists and clinicians. Requiring no programming knowledge, TOPAS provides all of the flexibility of Geant 4 .

Results: After 5 years of development followed by its initial release, TOPAS was subsequently expanded from its focus on proton therapy physics to incorporate radiobiology modeling. Next, in 2018, the developers expanded their user support and code maintenance as well as the scope of TOPAS towards supporting X-ray and electron therapy and medical imaging. Improvements have been achieved in user enhancement through software engineering and a graphical user interface, calculational efficiency, validation through experimental benchmarks and QA measurements, and either newly available or recently published applications. A large and rapidly increasing user base demonstrates success in our approach to dissemination of this uniquely accessible and flexible MC research tool.

Conclusions: The TOPAS developers continue to make strides in addressing the needs of the medical community in applications of ionizing radiation to medicine, creating the only fully integrated platform for four-dimensional simulation of all forms of radiotherapy and imaging with ionizing radiation, with a design that promotes inter-institutional collaboration.

\section{Purpose}

Improvements to medical applications of ionizing radiation require understanding how particles travel through material such as treatment heads, beam modifiers, detectors, imaging devices and the tissues and organs within the patient. The most precise calculations of such motion follow the Monte Carlo (MC) method. Yet MC's specialized computer programming techniques had limited its availability to specialists. The TOPAS TOol for PArticle Simulation [1], launched in 2009, is a breakthrough software project that struck down a usability barrier for MC simulations that was limiting radiation therapy research.

TOPAS is a wrapper for the all-particle MC toolkit Geant4 [2-4], initially developed for the high-energy physics community. TOPAS brings the sophisticated and reliable tools available in Geant4 into an experimentally validated and easy-to-use MC tool within reach of every medical physicist. Requiring little or no programming knowledge, TOPAS provides great flexibility. It enables cutting-edge research from detailed and accurate patient dose calculation to simulations of detectors under development for particle CT. TOPAS' modular, user- extensible design promotes collaboration among users and institutions.

First developed for proton therapy simulations, TOPAS is arguably a gold standard for proton therapy MC simulations. TOPAS was next expanded to radiobiology $[5,6]$. TOPAS has now become widely accepted in physics and biology, with 931 registered users at 342 institutions in 40 countries as of January 28,2020 . In addition, TOPAS has been licensed by several vendors of radiation therapy equipment and treatment planning software. TOPAS is part of the cadre of software tools supported by the National Cancer Institute program on Informatics Technology for Cancer Research with funding to support further development and sustainability [7].

The purpose of this paper is to bring readers up to date on recent developments in TOPAS, with emphasis on new capabilities and a selection of applications either newly available or published since the first general report [1].

\section{Materials and methods}

The code distribution presented here is TOPAS 3.2.p2. This version,

\footnotetext{
* Corresponding author at: UCSF Helen Diller Family Comprehensive Cancer Center, 1600 Divisadero Street, San Francisco, CA 94143, USA.

E-mail address: bruce.faddegon@ucsf.edu (B. Faddegon).
} 
Table 1

Example TOPAS parameters.

\begin{tabular}{|c|c|}
\hline Parameter & Function \\
\hline s:Ge/MyComponent/Type = "TsBox" & Declares new piece of geometry, a dividable box \\
\hline $\mathrm{i}: \mathrm{Ge} /$ MyComponent/HLZ $=1 . \mathrm{m}$ & Sets half-length of above box \\
\hline $\mathrm{i}: \mathrm{Ge} /$ MyComponent/ZBins $=10$ & Divides the box \\
\hline s:Ge/MyComponent/Material = "G4_WATER" & Sets material of the box \\
\hline s:Ge/MyComponent/Parent = "World" & Makes box a child of the World component \\
\hline $\mathrm{d}: \mathrm{Ge} /$ MyComponent/XTrans $=4 . \mathrm{cm}$ & Translates box from center of Parent \\
\hline $\mathrm{d}: \mathrm{Ge} /$ MyComponent/YRot $=30 \mathrm{deg}$ & Rotates box w.r.t parent coordinate system \\
\hline sv:Ph/Optical/Modules = 2 "g4optical" "g4em-standard_opt3" & Selects a particular set of Geant 4 physics modules \\
\hline s:Sc/DoseAtPhantom/Quantity = "DoseToMedium" & Creates a scorer of dose to medium \\
\hline s:Sc/DoseAtPhantom/Component = "MyComponent" & Attaches scorer to MyComponent \\
\hline sv:Sc/DoseAtPhantom /OnlyIncludeIfParticleOrAncestorInteractedInVolume = 1 "Propeller20/Leaf" & $\begin{array}{l}\text { Filters scorer to only trigger on cases where the scored particle or its } \\
\text { ancestor interacted in the given volume }\end{array}$ \\
\hline sv:Sc/DoseAtPhantom /OnlyIncludeIfInRTStructure = 2 "R_LUNG" "L_LUNG" & $\begin{array}{l}\text { If geometry was a DICOM rather than a simple box, limits sensitivity by } \\
\text { RTStruct to the two lungs }\end{array}$ \\
\hline
\end{tabular}

released in August 2019, contains pre-built versions of Geant4 10.5.p01 and the DICOM toolkit GDCM 2.6.8. The fundamental software architecture is unchanged from the original TOPAS, but many new features have been added. TOPAS is a $\mathrm{C}++$ application that wraps and extends the Geant4 software toolkit. A software application is a piece of code that one can download and run for a particular function. A software toolkit is a set of software pieces that can be assembled to create various applications. A toolkit user is expected to themselves be a software application developer. Whereas users of the native Geant4 need to write their own $\mathrm{C}++$ "User Applications" to exploit Geant4's flexible features, the TOPAS user does not need to write any $\mathrm{C}++$ code, but instead tailors their simulation by writing one or more "TOPAS Parameter Control Files," in an easy to learn ASCII file format.

For a detailed description of TOPAS features, see the original general paper [1] and additional details provided in this paper. The following are key features of TOPAS:

1. Uses the Monte Carlo method to simulate the passage of ionizing radiation through any complex geometry including medical instrumentation, detectors and the patient,

2. Handles complex motion through its innovative Time Feature System [8] and thus accommodates time-dependencies in modern therapies such as time-dependent particle beams and treatment head components, and they may involve patient motion (breathing, heartbeat, etc.).

3. Is based on a novel parameter control system designed to help reduce user errors.

4. Allows modeling of therapy and imaging devices without requiring programming experience.

5. Models any fundamental particle, including photons, electrons, protons and heavy ions.

6. Provides patient import from DICOM and other formats.

7. Is multithreaded (work is automatically spread and results collated over multiple CPUs).

8. Is highly extensible (expert users can write additional $\mathrm{C}++$ modules and share these with others). While TOPAS provides a large library of ready-made modules for geometry, scoring, filtering, etc., it also allows expert users to add their own extensions that go beyond these capabilities. The TOPAS Extensions Framework makes it trivial to share these modules with others.

9. Is designed to facilitate collaboration and sharing of simulation setups (inheritance mechanism of parameter files). Users may share setups and may modify them in ways that the setup's original author may not have anticipated.

The Geant 4 collaboration developed Geant 4 as an object-oriented software toolkit to achieve the key imperative of flexibility (such flexibility in large shared projects was a key motivation for the creation of the object oriented paradigm). In doing so, the Geant 4 collaboration knowingly placed a burden on other software developers to create their own software applications. For the initial users of Geant 4 this toolkit approach was a good fit. These users were large High Energy Physics collaborations each with sufficient workforce to delegate several team members to learn $\mathrm{C}++$ and then spend several years developing Geant4 applications specific to their experiments. The Geant4 collaboration always anticipated users in other fields would come together to create their own easier-to-use and more specialized Geant4-based applications.

The creation of a Geant4-based application requires the application developer to write at least five $\mathrm{C}++$ classes (main, geometry definition, physics list, primary particle generation, action initialization). For all but the first of these, Geant 4 provides a $\mathrm{C}++$ abstract class, while the user must provide a corresponding $\mathrm{C}++$ concrete class. Applications that exploit additional features of Geant4 may need to include many additional classes. The TOPAS collaboration has written these classes so that the end user does not need to. These key TOPAS classes interact with the rest of Geant4 through the $\mathrm{C}++$ design principle of being concrete instantiations of abstract classes. The TOPAS classes are written in such a way that they do not contain explicit instructions about what to build or how to run, but rather determine what to do at run time according to a set of user-supplied text files called TOPAS Parameter Control Files. The control system is described in the first TOPAS general paper [1]. Hundreds of TOPAS parameters are available to specify all aspects of the simulation, from geometry to physics settings, scoring options, variance reduction setups, time variation and more. Sample parameters are shown in Table 1. Up to date details are given in the TOPAS User Guide.

The user supplies the name of a single Control File as the one and only argument to the TOPAS executable. That file may in turn import additional Control Files. The Control Files may specify additional optional input to TOPAS such as DICOM files to define the patient, phase space files to define the source, and CAD files to specify some complex shapes. Output depends on the user's parameter settings, with a wide range of formats available to output scored quantities, from commaseparated-values to more compact binary representations, $\mathrm{xml}$ or root ntuple, DICOM dose, phase space files, etc.

Table 2 lists some of the key Geant 4 base classes, and the corresponding TOPAS concrete classes. Consider just the first of the Geant4 required classes, geometry specification. Consider a simple case: a user wants to define a geometry to be a cylinder of water placed at a given rotation and translation relative to the Cartesian coordinate system origin at the center of the simulated world of air. Fig. 1 shows just a portion of the $\mathrm{C}++$ code that a native Geant 4 user would write as their required concrete implementation of G4VUserDetectorConstruction. They would also have to write the other required Geant4 classes then compile and link their code against the Geant 4 toolkit. The TOPAS user 
Table 2

A selection of Geant4 base classes and the corresponding concrete TOPAS classes.

\begin{tabular}{lll}
\hline Function & Geant4 Base Class & Topas Class \\
\hline specify geometry & G4VUserDetectorConstruction & TsGeometryManager \\
specify physics processes to model & G4VUserPhysicsList & TsPhysicsManager \\
generate primary particles & G4VUserPrimaryGeneratorAction & TsSourceManager \\
initialize all user classes & G4VUserActionInitialization & TsActionInitialization \\
control per-event behavior & G4UserEventAction.hh & TsEventAction \\
control per-run behavior & G4MTRunManager & TsSequenceManager \\
control visualization & G4VisManager & TsGraphicsManager \\
control particle stack & G4UserStackingAction & TsStackingAction \\
control action per track & G4UserTrackingAction & TsTrackingAction \\
control action per track step & G4UserSteppingAction & TsSteppingsAction \\
persist information for scoring & G4VUserTrackInformation & TsTrackInformation \\
\hline
\end{tabular}

doing the same task writes the parameter control file shown as the inset in Fig. 1. There is nothing for them to compile or link (not even Geant4, as a copy of Geant4 is already prebuilt into TOPAS).

The TOPAS development team took an "engineering approach" to the overall design of TOPAS, meaning the system was designed by anticipating common categories of user mistakes and then building in protections against such mistakes. A few examples: 1) To avoid the likelihood that users would enter invalid values for a parameter, the TOPAS parameter control design adopts a concept from computer science called "strong typing", in which a parameter's type (string, boolean, integer, dimensioned value or un-dimensioned value) must be declared as part of the parameter definition. This allows the design to incorporate many consistency checks at startup. 2) To avoid the case where a user misunderstands the expected units for a dimensional parameter, there are no default units assumed, but rather the user is always required to include units in such parameters. 3) To avoid the likelihood that a user would accidentally lay out their parameters in the wrong order, or insert a new parameter into an existing file in the wrong location, the TOPAS design makes line order irrelevant within a parameter control file. 4) To avoid the case where a change to a parameter in a parameter control file supplied by one group (say the group within ones institution responsible for design of flat panel detectors) could adversely affect the behavior of a parameter control file supplied by another group (say the group responsible for the design of a linac treatment head within that institution), the control system forbids use of a parameter modified in one parameter file chain from use in another, parallel, parameter file chain.

TOPAS provides flexibility in scoring quantities. Table 3 lists the

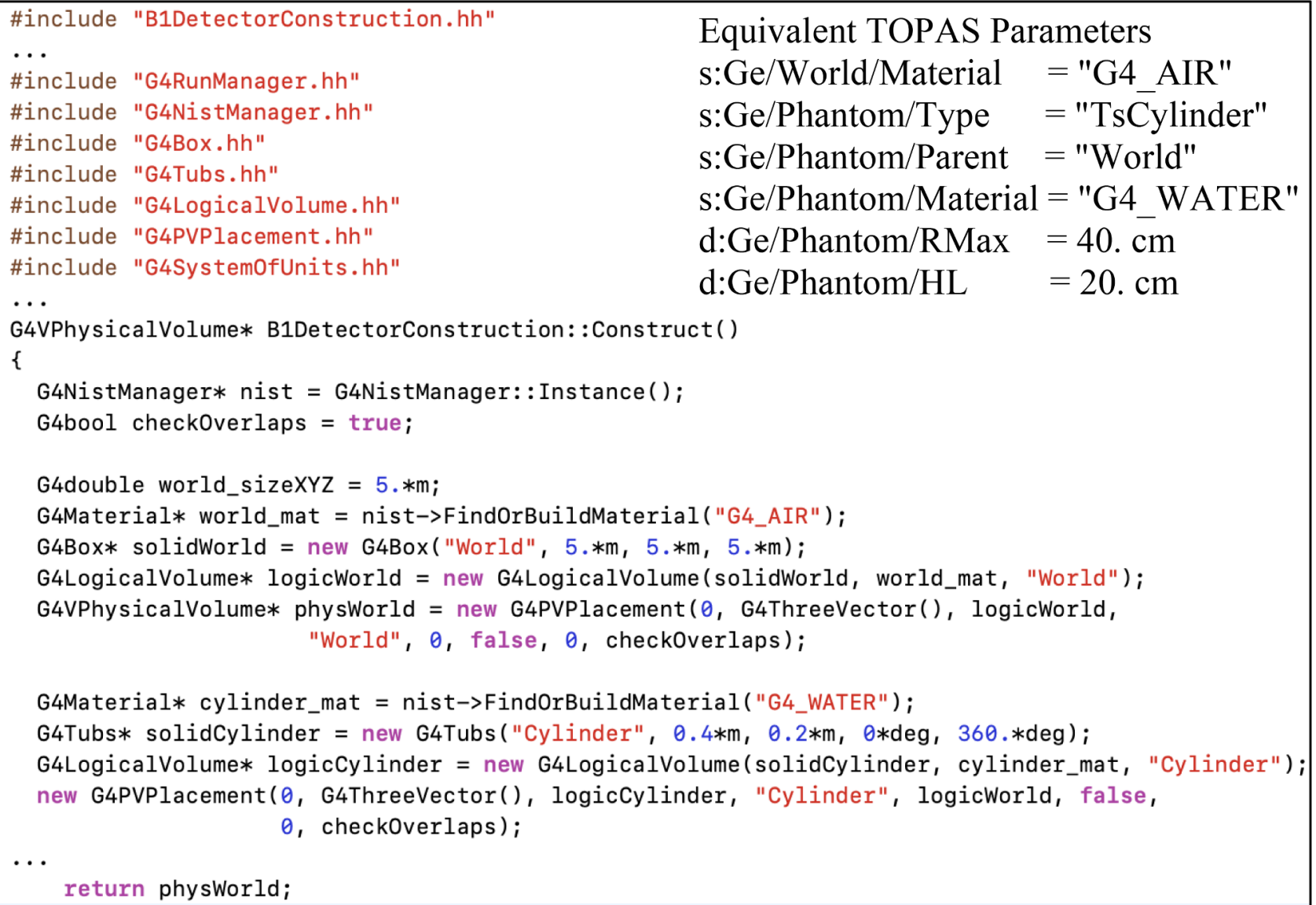

Fig. 1. A portion of the $\mathrm{C}++$ code that a user of native Geant 4 would need to write to define a simple setup of a cylinder of water centered in a world of air (with some details removed for space). Inset shows the equivalent full set of TOPAS parameters for this setup. 
Table 3

Built-in scorers.

\begin{tabular}{ll}
\hline Physical Quantity Scored & TOPAS parameter \\
\hline $\begin{array}{l}\text { Dose deposited in medium } \\
\text { Dose deposited in a Bragg-Gray water cavity }\end{array}$ & $\begin{array}{l}\text { DoseToMedium } \\
\text { DoseToWater } \\
\text { Dose deposited in a Bragg-Gray cavity }\end{array}$ \\
Energy deposited in medium & EnergyDeposit \\
Fluence & Fluence \\
Energy fluence & EnergyFluence \\
Count number of MC transportation steps & StepCount \\
Record optical photons to an ntuple fule & OpticalPhotonCount \\
Charge deposited (taken from PDG charge) & Charge \\
Charge deposited (taken from PreStepPoint) & EffectiveCharge \\
LET of primary and secondary protons & ProtonLET \\
Count of particles crossing surface & SurfaceTrackCount \\
Count of particles per surface area & SurfaceCurrent \\
Particle information on reaching surface: Energy, position, & PhaseSpace \\
\multicolumn{1}{c}{ direction, etc } & \\
\hline
\end{tabular}

current set of built-in scorers. Table 4 lists a subset of current built-in filters that can be applied to these scorers. Expert users may also write their own scorers and filters as $\mathrm{C}++$ extensions. Additional features of TOPAS scoring include the following:

1. Scorers default to use same binning as the component they are applied to, but may also be set to use different binning. So, for example, a water phantom built as $10 \times 10 \times 10$ may be scored as $15 \times 15 \times 10$.

2. The same quantity may be binned several different ways at the same time. So, for example, the patient dose may be simultaneously binned in the full CT resolution and at a reduced resolution used by the treatment planning system.

3. Any number of filters may be applied in series

4. Scored results may be accumulated then output per overall session, per history, or per run (a user-defined subset of a full simulation session, for example, a set of histories for which all geometry parameters, electric and magnetic field parameters and particle source parameters are constant).

5. All scored quantities can optionally include statistical information (variance, standard deviation, $\mathrm{min} / \mathrm{max}$ values, etc.).

6. Scorers may be gated, that is, made active or inactive at various points during the simulation.

\section{Results}

\subsection{User enhancement}

Extensions Framework: TOPAS has always provided the ability for advanced users who want to write $\mathrm{C}++$ to link their own $\mathrm{C}++$ into

Table 4

A subset of available scoring and particle generation filters.

\footnotetext{
Particle generation (primary vs secondary)

Charge

Atomic Mass

Atomic Number

Initial Kinetic Energy

Initial Momentum

Origin process (scattering, Bremsstrahlung, etc.)

Ancestor process (above but also considering particle's ancestors)

Particle name (gamma, e-, proton)

Ancestor named (above but also considering particle's ancenstors)

Origin component (geometry component the particle originated in)

Interacted in component

Traversed component

Material (only score if in the given material)

RT Structure (only score if in the given DICOM RT Structure set)
}

the existing TOPAS, however this "extensions framework" has been significantly enhanced since the original TOPAS release. Extension classes can now add new Geometry Components, Imaging-to-Material Converters (in place of the standard TOPAS method of Hounsfield Unit conversion), Magnetic, Electric and Electromagnetic Field Definitions, Filters, Particle Sources, Scorers, Outcome Models, Geant4 Physics Lists and Geant4 Physics Modules. Additional hooks have been added such that users can have their own code called at various times in the simulation process (Begin Session, Begin Run, Begin History, End History, End Run and End Session). The overall build system has been redesigned so that CMake automatically writes the "glue code" to attach user extensions into the rest of TOPAS. Users simply put all of their desired extension classes into a folder (or hierarchy of folders), and CMake weaves these extensions into a new TOPAS executable, whether extensions were written by the user or obtained from others.

GUI: A Graphical User Interface (GUI) has been added as a new option. TOPAS Parameter Control Files remain at the heart of TOPAS, clearly specifying and "locking down" every parameter of the simulation. The new, optional GUI provides a way to quickly visualize the simulation setup and rapidly experiment with parameter changes. The main window of the GUI provides interactive OpenGL graphics allowing the user to zoom and rotate geometry and particle tracks and to click on geometry or tracks to interrogate details. A Parameter Control Widget, lets the user modify any parameter (such as changing the size or orientation of a geometry component) and immediately see the results in the main visualization window. Geant4 geometry overlap checking has been revised for this GUI so that, rather than an overlap being a fatal program error, overlaps are simply noted, and the user can then adjust the geometry to remove them. A simulation run can only be started after all overlaps are resolved. Additional buttons in the GUI allow the user to add and adjust new geometry components, particle sources or scorers, and to start fresh simulation runs after any changes. Finally, the user can save the new setup, creating a new TOPAS parameter control file that incorporates the changes the user has made through the GUI. A user can subsequently start a new simulation from the saved setup. Standard usage is to work within the GUI until a short simulation looks reasonable, then save the setup and run a much longer simulation with the saved parameter file on a non-GUI cluster or cloud simulation to achieve the required precision in the calculated quantities.

New Geant4 capabilities: TOPAS has evolved to stay up to date with the latest Geant4 releases (moving gradually through the releases from Geant4 9.5 to Geant4 10.5.p1). The improvements from one Geant4 version to another are too numerous to mention, but TOPAS always offers the latest Geant4. The most significant Geant4 improvement over the lifetime of TOPAS was that, at version 10.0, Geant4 became the first (and still only) fully flexible MC transport code to enable multithreading. Multi-threading allows a software application to spread work over multiple CPU cores and multiple threads per core, so that a single user job can access the full capabilities of today's multi-core computer architectures. Without this feature, the user must run separate simulation jobs on each core and then hand collate results from these separate jobs. Complex Geant4-based applications still require significant redesign to exploit Geant4's multithreading capabilities. TOPAS completed such a redesign in TOPAS 2.0 (released November 2015), such that any TOPAS user can multi-thread their simulation by simply adjusting a single TOPAS parameter (Ts/NumberOfThreads). Every TOPAS feature supports multithreading.

New Operating Systems: TOPAS has expanded the selection of prebuilt TOPAS versions to support new system architectures. The latest TOPAS runs on any Mac OS from 10.12 onwards, Debian 8, 9 and 10 (and variants such as Ubuntu), CentOS6, 7 and 8 (and variants such as RHEL).

Continuing user support: TOPAS developers have continually engaged with their user community through an online forum that in January 2020 had 632 topics, each topic averaging 7 posts. User- 

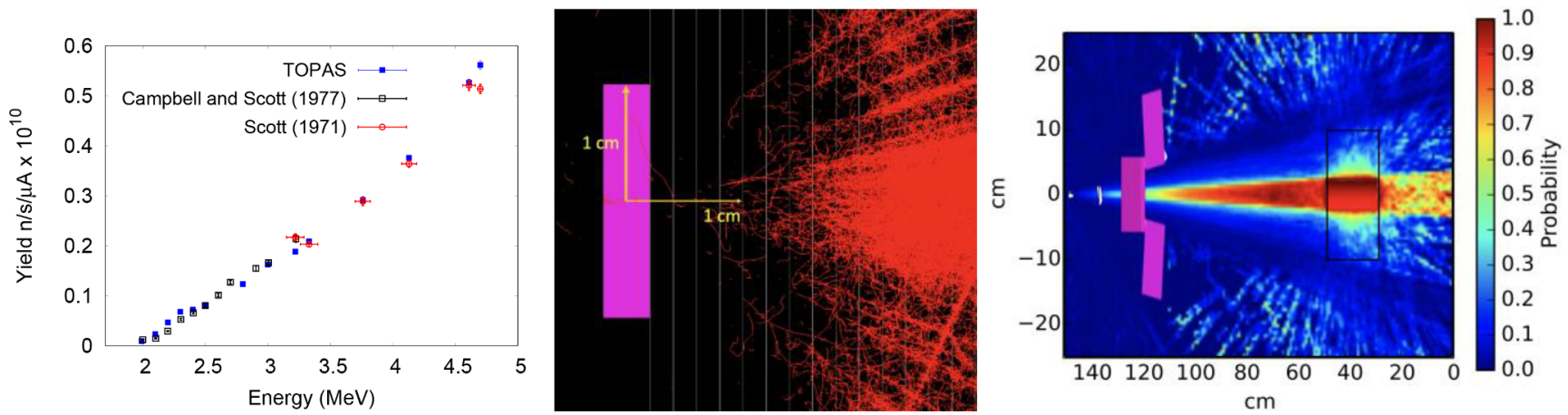

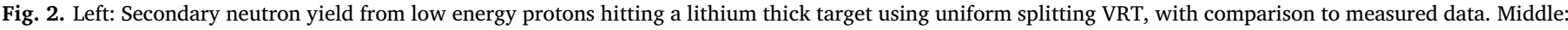

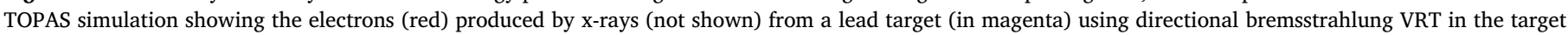

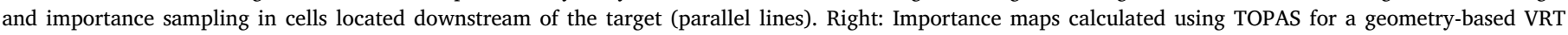

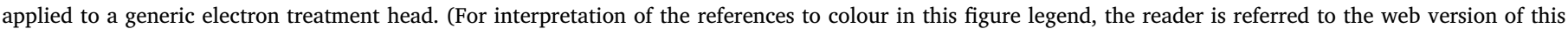
article.)

reported bugs have been addressed and new features added at a steady rate.

\subsection{Efficiency improvements}

TOPAS provides a wide, extendible yet easy-to-use suite of variance reduction techniques (VRTs). Examples are depicted in Fig. 2. TOPAS has a geometrically based uniform particle splitting technique designed for passive-scattering proton therapy $[9,10]$, photon interaction forcing [11], cross-section enhancement of electromagnetic processes [12], geometrical importance sampling and weight window techniques. Approximate efficiency improving techniques are also available: electron range rejection, region-based cuts to secondary particle production, and terminating particles in user-specified volumes. Several VRTs may now be easily used in the same simulation with minimum programming effort. Automatically generated geometrical grids to configure importance maps automate the configuration of importance sampling. The characterization of the VRTs implemented in TOPAS is an ongoing task.

Investigation of cost-effective hardware-accelerated MC is an ongoing effort in TOPAS. TOPAS contributes to an international collaboration to evaluate the feasibility of increasing computational speed through Field Programmable Gated Arrays (FPGAs) [13].

\subsection{Validation}

Validation with continued regression testing is critical to determine and maintain the accuracy of simulations. TOPAS has previously been validated for proton therapy with measurements in the MGH beam line [14]. TOPAS developers are active in the Geant4 Medical Simulation Benchmarking Group (G4MSBG). The group has collaborated to produce an extensive list of benchmarks of Geant4 relevant to medical uses of ionizing radiation and to compare simulation results with measurement or theory using the Geant4 regression testing system [15]. Current results are available at https://geant-val.cern.ch. The TOPAS team is actively participating in the G4MSBG benchmarking effort and have contributed the following experimental benchmarks:

$\mathrm{X}$-ray yield from thick targets [16] and electron scatter from moderately thin foils [17], both for materials and beam energies relevant to radiotherapy, a proton Bragg curve of known beam energy measured with sub-millimeter accuracy [18] and a comparison of Geant4 calculations to experimental benchmarks of neutron yield from protons and carbon ions incident of thick targets.

\subsection{Applications}

Since its initial release in 2012, TOPAS has seen wide acceptance in a broad range of applications, with 346 citations of the general paper
[1] in Google Scholar as of February 3, 2020. The following is a selection of newly available and recently published applications.

Microdosimetric extension: Cellular response to ionizing radiation is highly dependent on energy deposited by the incident particle on the microdosimetric scale, and could determine the relative biological effectiveness (RBE) in proton and ion therapy. MC simulations of energy deposition distributions on a micro- or nano-scale, combined with recent advances in micro-detector technology have become an important tool in radiotherapy. An extension to TOPAS provides users the functionality to simulate microdosimetric spectra and determine RBE with a biological weighting function approach or with the microdosimetric kinetic model [19].

DICOM interface: MC simulations can serve as an independent dose calculation over the course of radiotherapy treatment, from the planning to quality assurance (QA) stages. A DICOM-RT Ion interface developed and integrated into TOPAS can convert DICOM format treatment information into TOPAS setups. This DICOM capability allows TOPAS to be used, for example, to calculate dose distributions for individual patients and the QA of those patients based on the treatment plan information from a radiotherapy treatment planning system. The interface supports Ion Plan (RTIP) and Ion Beams Treatment Record (RTIBTR) so that TOPAS can calculate patient dose as well as water phantom dose with a planned and/or a delivered fluence map. Depictions of independent dose calculations in proton therapy using the TOPAS DICOM interface are shown in Fig. 3.

Brachytherapy: A new TOPAS particle source model, the "Volumetric Source," has extended TOPAS to brachytherapy applications. In this mode, the user specifies the name of a radioactive material within a given source component, and TOPAS then starts particles from randomly sampled points within this active material. An example included in the TOPAS distribution, shown in Fig. 4, demonstrates HDR brachytherapy treatment with a Varian source wire moving through dwell positions in a simulated gynecological applicator (using TOPAS 4D capabilities to move the wire). The Geant4 physics at the heart of TOPAS has already been validated for brachytherapy [20,21]. The G4MSBG group has added a benchmark against published measurements of the 192Ir Flexisource [22]. Additional validation of this new brachytherapy capability in TOPAS by comparison to results from other codes and experiment data is currently under way by other groups.

Detectors in particle therapy: With its advanced capabilities of simulating complex source and geometry details and variations of these details in time, TOPAS is an ideal tool to assist in the design and model ionizing radiation detectors through accurate and detailed calculations of critical quantities such as detector efficiency and response. TOPAS can calculate absorbed dose to water in a reference water cavity as well as absorbed dose in air cavities representative of plane-parallel or cylindrical ionization chambers [23]. TOPAS has proven instrumental in 

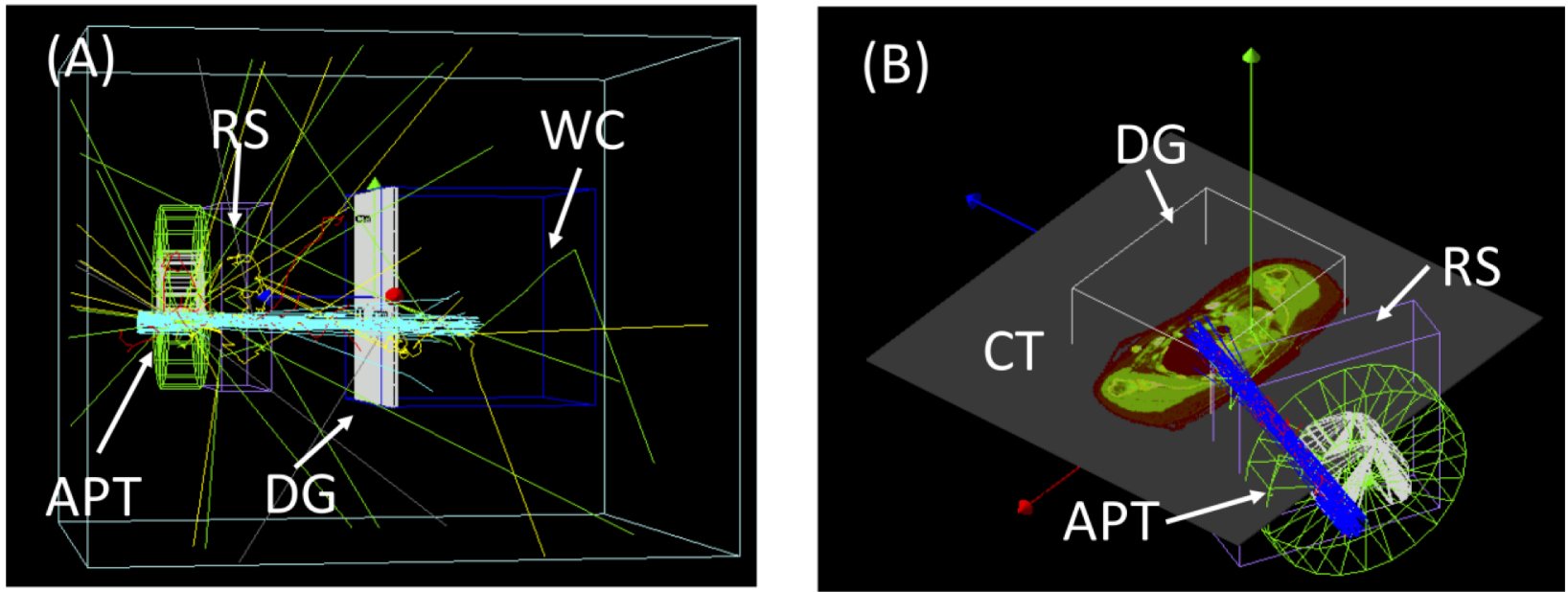

Fig. 3. Examples of Independent dose calculations using the TOPAS DICOM interface, (A) Patient specific QA and (B) a patient dose recalculation. The interface sets up patient specific geometries, such as range shifter (RS), aperture (APT), water phantom (WC), and dose-grid (DG), including translational and rotational movements.
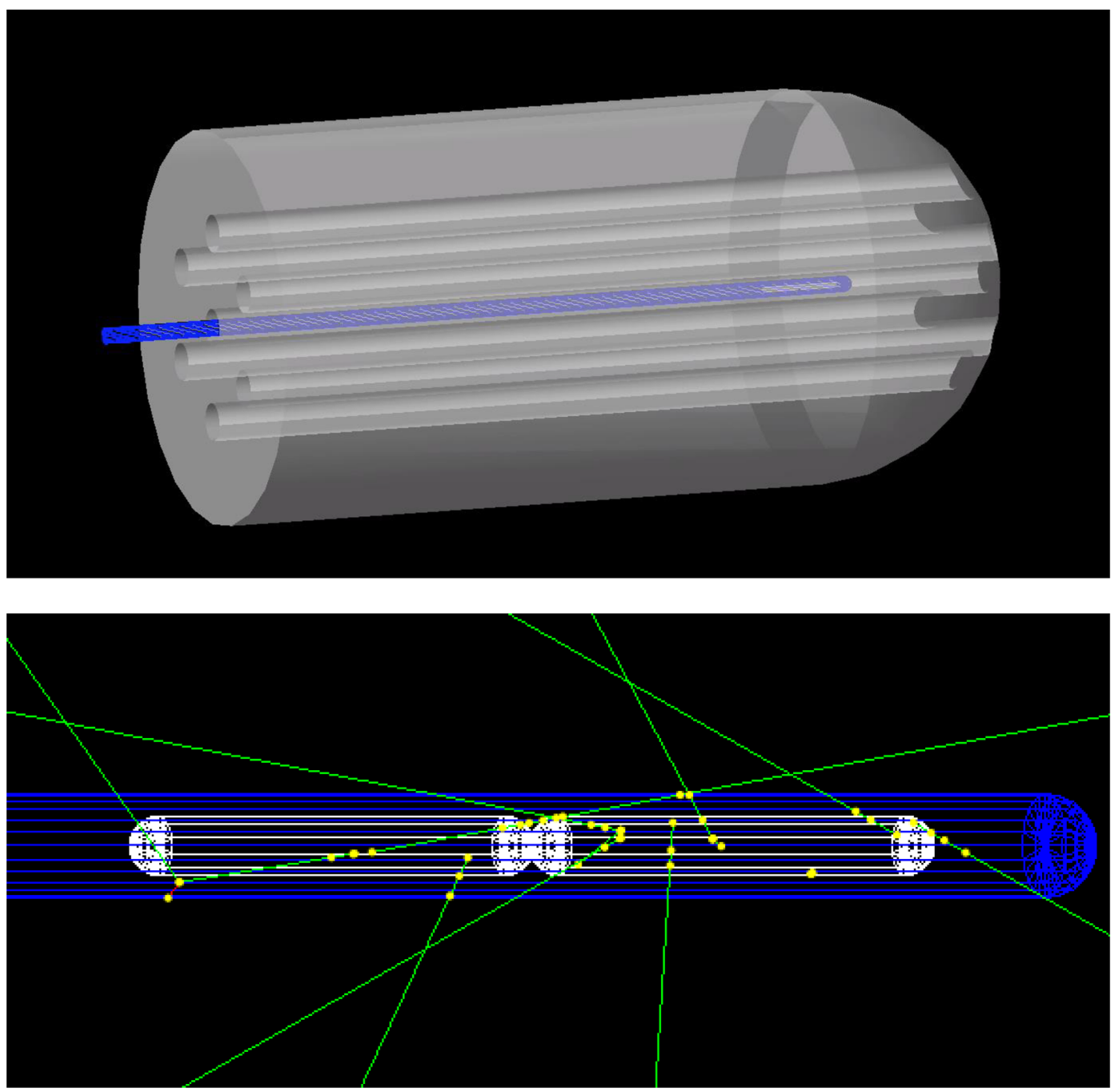

Fig. 4. TOPAS brachytherapy example. Top: HDR brachytherapy treatment with a Varian source wire at one dwell position in a simulated gynecological applicator. Bottom: Close-up of the wire (blue) showing gamma-rays (green) emitted from randomly sampled positions within the Ir192 source capsules (white). (For interpretation of the references to colour in this figure legend, the reader is referred to the web version of this article.) 
development of a variety of detectors beyond radiation dosimetry. Recent applications include time-resolved diode dosimetry (proposed for in vivo proton range verification) to calibrate the detector by simulating water-equivalent beam path lengths in tissue [24]. TOPAS simulations have also been used in the development of scanners and the associated calibration and reconstruction methods for proton [25] and helium CT [26] (promising methods for mitigating range uncertainty). A TOPAS study of the impact of secondary fragments to image quality of helium ion imaging [27] led to improved methodology for singleevent proton CT by removing nuclear interaction events within the energy/range detector [28]. TOPAS has also been used to study proton radiography [29] and X-ray fluorescence spectroscopy [30,31]. Prompt gamma (PG) photons produced by proton-nuclear interactions inside the patient may be detected outside the body to monitor the proton range in the patient during treatment. Time-dependent simulations with TOPAS have been used to demonstrate proton range verification with a single measurement point and a simple detector configuration [32]. A TOPAS study investigated medium and detector properties that influence accuracy in PG range measurement [33].

Patient dose and dose effect calculations: MC is often used to calculate patient dose distributions using CT $[34,35]$. This includes evaluation of dose calculation methods in clinical treatment planning systems (TPS) for effects such as patient heterogeneities and resulting range uncertainties due to simplified modeling of multiple-Coulomb scattering at high density tissue gradients in the TPS [36,37]. Differences between TPS and MC for complete treatments have been investigated by multiple groups [38-46]. Highly efficient MC systems are being developed for dedicated patient dose calculations. TOPAS has been used to commission such systems [47-51].

MC methods provide information beyond dose, such as linear energy transfer (LET) distributions [52-54]. Increasing density of energy depositions, i.e. increasing LET, is associated with a radiation therapy modality's increased relative biological effect (RBE). TOPAS offers an interface to many RBE models [55] and has been used in several studies on RBE effects in proton therapy patients [53,54,56,57]. To model biological effect in patients, TOPAS includes tumor control probability (TCP) and normal tissue complication probability (NTCP) models [9]. A separate effort, TOPAS-nBio [58], looks at radiation effects at the cellular scale.

Dissemination

TOPAS has reached a wide user base through a combination of workshops, online tutorials and online courses. A non-profit organization, TOPAS MC Inc., disseminates the software through the website topasmc.org. at no charge to users from non-profit institutions worldwide. Every TOPAS user is required to have their own single-user TOPAS license and to attend a $90 \mathrm{~min}$ in-person online tutorial session given by a TOPAS developer. This in-person session has ensured that new users share a common, baseline understand of the software and helped TOPAS develop a robust and friendly community in the TOPAS user forum. In-person tutorials have also been given, in concert with various medical physics conferences. A course on MC simulations for radiation therapy with TOPAS was held at the Autonomous University of Puebla, Mexico in October of 2018 [59]. In-person courses approved for CAMPEP credit are also given on an annual basis, the first held in March of 2019 for a full day at UCSF [60].

\section{Conclusion}

TOPAS supports simulation of all ionizing particle types through complex geometries found in medical applications including treatment heads, beam modifiers, patients, and detectors. Since June 2018, the National Cancer Institute initiative in Informatics Technology for Cancer Research is facilitating TOPAS applications beyond proton therapy. TOPAS has demonstrated potential for broad impact. While sustaining and advancing TOPAS for its initial user base, the TOPAS collaboration has innovated to address emergent needs from across the cancer research and treatment continuum, becoming the only integrated platform for advanced radiotherapy to support multi-modality treatments, inter-modality comparisons and the full range of image guidance.

\section{Acknowledgments}

TOPAS development has been supported by the following research grants from the USA National Institutes of Health: R01 CA140735 and U24 CA215123.

\section{References}

[1] Perl, Shin J, Schümann J, Faddegon B, Paganetti H. TOPAS - an innovative proton Monte Carlo platform for research and clinical applications. Med Phys. 2012;39:6818-37. PMID: 23127075.

[2] Agostinelli S, et al. GEANT4 - a simulation toolkit. Nucl Instruments Methods Phys Res Sect A Accel Spectrometers, Detect Assoc Equip 2003;506:250-303.

[3] Allison J, et al. Geant4 developments and applications. IEEE Trans Nucl Sci 2006;53(1):270-8.

[4] Allison J, et al. Recent developments in GEANT4. Nucl Instruments Methods Phys Res Sect A Accel Spectrometers, Detect Assoc Equip 2016;835:186-225.

[5] Ramos-Méndez Jose, Perl Joseph, Schuemann Jan, Shin Jungwook, Paganetti Harald, Faddegon Bruce. A framework for implementation of organ effect models in TOPAS with benchmarks extended to proton therapy. Phys Med Biol 2015;60(13):5037-52. PMID: 26061583.

[6] Lisa Polster, Jan Schuemann, Ilaria Rinaldi, Lucas Burigo, Aimee Louise McNamara, Robert D Stewart, Andrea Attili, David J. Carlson, Alejandro Carabe-Fernadez, Bruce Faddegon, Joseph Perl, Harald Paganetti, Extension of TOPAS for the simulation of proton radiation on molecular and cellular endpoints, Phys Med Biol, 2015 Jun 10;60(13):5053-5070, PMID: 26061583.

[7] https://projectreporter.nih.gov/project_info_description.cfm?aid $=9506198 \&$ icde $=42307512 \&$ ddparam $=\& d d v a l u e ~=\&$ ddsub $=\& \mathrm{cr}=1 \& \mathrm{csb}=$ default $\& \mathrm{cs}=$ ASC \&pball.

[8] Shin J, Perl J, Schümann J, Paganetti H, Faddegon BA. A modular method to handle multiple time-dependent quantities in Monte Carlo simulations. Phys Med Biol 2012;57(11):3295-308. PMID: 22572201.

[9] Ramos-Mendez JA, Perl J, Faddegon B, Schuemann J, Paganetti H. Geometrical splitting technique to improve the computational efficiency in Monte Carlo calculations for proton therapy. Med. Phys. 2013;40(4). 041718-1-10, PMID: 23556888

[10] Ramos-Méndez Jose, Perl Joseph, Faddegon Bruce, Schuemann Jan, Shin Jungwook, Paganetti Harald. Improved efficiency in Monte Carlo simulation for passive-scattering proton therapy. Phys Med Biol 2015;60(13):5019-35. PMID 2606145.

[11] Bielajew AF, Rogers DWO, 1988 Variance-reduction techniques in Monte Carlo transport of electrons and photons ed T M Jenkins, W R Nelson and A Rindi (Ettore Majorana International Science Series) p 407.

[12] Ali ESM, Rogers DWO. Efficiency improvements of x-ray simulations in EGSnrc user-codes using bremsstrahlung cross-section enhancement (BCSE). Med Phys 2007;34:2143.

[13] Hemant Darbari, C. Sajish, U.B. Sonavane, Vivek Gavane, Abhay Deshpande, Tanuja Dixit, Rajesh Harsh, Rajendra Badwe, G.K. Rath, Siddhartha Laskard, Bruce Faddegon, Joseph Perl, Harald Paganetti, Jan Schuemann, Anil Srivastava, Ceferino Obcemea, Asheet K. Nath, Ashok Sharma, Jeffrey Buchsbaum, Monte Carlo Processing on a Chip (MCoaC)-preliminary experiments toward the realization of optimal-hardware for TOPAS/Geant4 to drive discovery, Physica Medica, 64: $166-173,2019$.

[14] Testa M, Schümann J, Lu H-M, Shin J, Faddegon B, Perl J, et al. Experimental validation of the TOPAS Monte Carlo system for passive scattering proton therapy. Med Phys 2013;40(12):121719. PMID: 24320505.

[15] Arce P, Bolst D, Cutajar D, Guatelli S, Sakata D, Bordage M-C, Cirrone P, Cuttone G, Pandola L, Petringa G, Cortes-Giraldo MA, Quesada JM, Desorgher L, Dondero P, Mantero A, Dotti A, Wright D, Faddegon B, Ramos-Mendez J, Fedon C, Incerti S, Ivanchenko V, Konstantinov D, Latyshev G, Kyriakou I, Mancini-Terraccciano C, Maire M, Novak M, Omachi C, Toshito T, Perales A, Perrot V, Romano F, Sarmiento L, Sasaki T, Sechopoulos I, Simpson EC. Development of a benchmarking system for validation and regression testing of Geant4 for medical physics. Med Phys 2019. Under review.

[16] Bruce A Faddegon, Makoto Asai, Joseph Perl, Carl Ross, Josep Sempau, Jane Tinslay, and Francesc Salvat, Benchmarking of Monte Carlo simulation of bremsstrahlung from thick targets at radiotherapy energies, Med Phys, 35(10):4308-4317, 2008, PMID: 18975676, PMCID: PMC2736754.

[17] Bruce A Faddegon, Iwan Kawrakow, Yuri Kubyshin, Joseph Perl, Josep Sempau, and Laszlo Urban, The accuracy of EGSnrc, Geant4 and PENELOPE Monte Carlo systems for simulation of electron scatter in external beam radiotherapy, Phys Med Biol, 54:6151-6163, 2009, PMID: 19779217.

[18] Faddegon Bruce A, Shin J, Castenada Carlos M, Ramos-Mendez Jose, Daftari Inder K. Experimental depth dose curves of a $67.5 \mathrm{MeV}$ proton beam for benchmarking and validation of Monte Carlo simulation. Med Phys 2015;42(7):4199-212. PMCID PMC4474955.

[19] Hongyu Zhu, Yizheng Chen, Wonmo Sung, Aimee L. McNamara, Linh T. Tran, Lucas N. Burigo, Anatoly B. Rosenfeld, Junli Li, Bruce Faddegon, Jan Schuemann, Harald 
Paganetti, "The microdosimetric extension in TOPAS: development and comparison with published data," In press, PMB, May, 2019.

[20] Enger Shirin A, Landry Guillaume, D'Amours Michel, Verhaegen Frank, Beaulieu Luc, Asai Makoto, Perl Joseph. Layered mass geometry: a novel technique to overlay seeds and applicators onto patient geometry in Geant4 brachytherapy simulations. Phys Med Biol 2012;57:6269.

[21] Afsharpour H, Landry G, D'Amours M, Enger S, Reniers B, Poon E, et al. ALGEBRA: ALgorithm for the heterogeneous dosimetry based on GEANT4 for BRAchytherapy. Phys Med Biol 2012;57:3273.

[22] Granero D, Perez-Calatayud J, Casal E, Ballester F, Venselaar J. A dosimetric study on the high dose rate exisource. Med Phys 2006;33(12):4578-82.

[23] Baumann, et al. Comparison of PENH, FLUKA and Geant4/TOPAS for absorbed dose calculations in air cavities representing ionization chambers in high-energy photon and proton beams. Med Phys 2019. PMID: 31350915.

[24] Toltz et al., Time-resolved diode dosimetry calibration through Monte Carlo modeling for in vivo passive scattered proton therapy range verification. J Appl Clin Med Phys. 2017 Nov; 18(6): 200-205.

[25] Piersimoni P, Ramos-Mendez J, Geoghean T, Bashkirov VA, Schulte RW, Faddegon BA. The effect of beam purity and scanner complexity on proton CT accuracy. Med Phys 2017:44(1):284-98.

[26] Pierluigi Piersimoni, Bruce A. Faddegon, José Ramos Méndez, Reinhard W. Schulte, Lennart Volz, Joao Seco, "Helium CT: Monte Carlo simulation results for an ideal source and detector with comparison to proton CT," Med Phys 45(7):3264-74, July, 2018.].

[27] Volz L, Piersimoni P, Bashkirov VA, Brons S, Collins-Fekete CA, Johnson RP, Schulte RW, Seco J., The impact of secondary fragments on the image quality of helium ion imaging, Phys Med Biol, 2018 Oct 2;63(19):195016. doi: 10.1088/1361-6560/ aadf25. PMID: 30183679.

[28] Volz L, Piersimoni P, Johnson RP, Bashkirov VA, Schulte RW, Seco J., Improving single-event proton CT by removing nuclear interaction events within the energy/ range detector, Phys Med Biol. 2019 Aug 1;64(15):15NT01. doi: 10.1088/13616560/ab2671. PMID: 31158820.

[29] Huo W, Zwart T, Cooley J, Huang K, Finley C, Jee KW, et al. A single detector energy-resolved proton radiography system: a proof of principle study by Monte Carlo simulations. Phys Med Biol 2019;64(2):025016.

[30] Dunning CAS, Bazalova-Carter M. Optimization of a table-top x-ray fluorescence computed tomography (XFCT) system. Phys Med Biol 2018;63(23):235013.

[31] Bazalova-Carter M. Proton-induced x-ray fluorescence CT imaging. Med Phys. 2015 Feb;42(2):900-7.

[32] Testa M, Min CH, Verburg JM, Schümann J, Lu HM, Paganetti H. Range verification of passively scattered proton beams based on prompt gamma time patterns. Phys Med Biol 2014;59:4181-95.

[33] Janssen FM1, Landry G, Cambraia Lopes P, Dedes G, Smeets J, Schaart DR, Parodi K, Verhaegen F. (2014) Factors influencing the accuracy of beam range estimation in proton therapy using prompt gamma emission, Phys Med Biol 59, 4427-41.

[34] Indrin J. Chetty, Bruce Curran, Joanna E. Cygler, John J. DeMarco, Gary Ezzell, Bruce A. Faddegon, Iwan Kawrakow, Paul J. Keall, Helen Liu, Charlie Ma, D. W. O. Rogers, Jan Seuntjens, Daryoush Sheikh-Bagheri, Jeffrey V. Siebers. Report of the AAPM Task Group No. 105: Issues associated with clinical implementation of Monte Carlo-based photon and electron external beam treatment planning, Med Phys 34:4818-4853, 2007, PMID: 18196810

[35] Schuemann J, et al. Efficient voxel navigation for proton therapy dose calculation in TOPAS and Geant4. Phys Med Biol 2012;57(11):3281-93.

[36] Schuemann J, Dowdell S, Grassberger C, et al. Site-specific range uncertainties caused by dose calculation algorithms for proton therapy. Phys Med Biol 2014;59:4007.

[37] Flatten V, Baumann K-S, Weber U, et al. Quantification of the dependencies of the Bragg peak degradation due to lung tissue in proton therapy on a CT-based lung tumor phantom. Phys Med Biol 2019;64:155005.

[38] Schuemann J, Giantsoudi D, Grassberger C, et al. Assessing the clinical impact of approximations in analytical dose calculations for proton therapy. Int J Radiat Oncol Biol Phys 2015.

[39] Geng C, Daartz J, Tin Cheung KL, et al. Dosimetric Uncertainties and Their Impact on Treatment Planning in Stereotactic Proton Radiosurgery. Int J Radiat Oncol Biol Phys 2016;96:E618.

[40] Liu H, Li Z, Slopsema R, et al. TOPAS Monte Carlo simulation for double scattering proton therapy and dosimetric evaluation. Phys Med 2019;62:53-62.

[41] Huang S, Kang M, Souris K, et al. Validation and clinical implementation of an accurate Monte Carlo code for pencil beam scanning proton therapy. J Appl Clin Med Phys 2018;19:558-72.

[42] Shin W-G, Testa M, Kim HS, et al. Independent dose verification system with Monte Carlo simulations using TOPAS for passive scattering proton therapy at the National Cancer Center in Korea. Phys Med Biol 2017;62:7598-616.

[43] Lin L, Huang S, Kang M, et al. A benchmarking method to evaluate the accuracy of a commercial proton monte carlo pencil beam scanning treatment planning system. $J$ Appl Clin Med Phys 2017;18:44-9.

[44] Fracchiolla F, Lorentini S, Widesott L, et al. Characterization and validation of a Monte Carlo code for independent dose calculation in proton therapy treatments with pencil beam scanning. Phys Med Biol 2015;60:8601-19.

[45] Hartman J, Kontaxis C, Bol GH, et al. Dosimetric feasibility of intensity modulated proton therapy in a transverse magnetic field of 1.5 T. Phys Med Bio 2015;60:5955-69.

[46] Geng C, Moteabbed M, Seco J, et al. Dose assessment for the fetus considering scattered and secondary radiation from photon and proton therapy when treating a brain tumor of the mother. Phys Med Biol 2016;61:683-95.

[47] Huang S, Souris K, Li S, et al. Validation and application of a fast Monte Carlo algorithm for assessing the clinical impact of approximations in analytical dose calculations for pencil beam scanning proton therapy. Med Phys 2018;45:5631-42.

[48] Giantsoudi D, Schuemann J, Jia X, et al. Validation of a GPU-based Monte Carlo code ( $\mathrm{gPMC}$ ) for proton radiation therapy: clinical cases study. Phys Med Biol 2015;60:2257-69.

[49] Bueno M, Paganetti H, Duch MA, Schuemann J. An algorithm to assess the need for clinical Monte Carlo dose calculation for small proton therapy fields based on quantification of tissue heterogeneities. Med Phys 2013;40(8):081704https://doi. org/10.1118/1.4812682.

[50] Tseung HWC, Ma J, Beltran C. A fast GPU-based Monte Carlo simulation of proton transport with detailed modeling of nonelastic interactions. Med Phys 2015;42:2967-78

[51] Qin N, Botas P, Giantsoudi D, et al. Recent developments and comprehensive evaluations of a GPU-based Monte Carlo package for proton therapy. IOP Publishing; 2016.

[52] Granville DA, Sawakuchi GO. Comparison of linear energy transfer scoring tech niques in Monte Carlo simulations of proton beams. Phys Med Biol 2015;60:N283-91.

[53] Giantsoudi D, Grassberger C, Craft D, et al. Linear energy transfer-guided optimization in intensity modulated proton therapy: feasibility study and clinical potential. Int J Radiat Oncol Biol Phys 2013;87:216-22.

[54] Giantsoudi D, Sethi RV, Yeap BY, et al: Incidence of CNS Injury for a Cohort of 111 Patients Treated With Proton Therapy for Medulloblastoma: LET and RBE Associations for Areas of Injury. Int J Radiat Oncol*Biol*Phys 95:287-296, 2016.

[55] Polster L, Schuemann J, Rinaldi I, et al. Extension of TOPAS for the simulation of proton radiation effects considering molecular and cellular endpoints. Phys Med Biol 2015;60:5053-70.

[56] Underwood T, Giantsoudi D, Moteabbed M, et al. Can we advance proton therapy for prostate? considering alternative beam angles and relative biological effectiveness variations when comparing against intensity modulated radiation therapy. Int J Radiat Oncol*Biol*Phys 2016;95:454-64.

[57] Underwood TSA, Grassberger C, Bass R, et al. Asymptomatic late-phase radiographic changes among chest-wall patients are associated with a proton RBE exceeding 1.1. Int J Radiat Oncol Biol Phys 2018;101:809-19.

[58] Schuemann J, McNamara AL, Ramos-Méndez J, et al. TOPAS-nBio: an extension to the TOPAS simulation toolkit for cellular and sub-cellular radiobiology. Radiat Res 2019;191:125-38.

[59] http://fisicamedica.mx/convocatorias/montecarlo2018.

[60] http://www.topasmc.org/home/topas-course-march-8th-2019. 